\title{
VI. Königlich-westphälische Wappen zwischen Aneignung und Angriff
}

Napoleon selbst betrachtete das königlich-westphälische Wappen, das im Dezember 1809 mit einem neuen Orden geschaffen worden war, mit Skepsis und äußerte seinem Bruder gegenüber: »il y a bien des bêtes dans cet ordre « ${ }^{1}$. Tatsächlich vereinte das Wappen des neuen Staates den napoleonischen Adler mit dem braunschweigischen und dem hessischen Löwen, dem steigenden Pferd Hannovers und weiteren Wappentieren einzelner Teilgebiete, als Wahrzeichen für die Verschmelzung der Kleinstaaten, die das Königreich Westphalen bildeten ${ }^{2}$. Wie empfanden die Einwohner Westphalens ihr neues Wappenbild?

$\mathrm{Zu}$ den ersten Amtshandlungen der neuen westphälischen Herrschaft gehörte der Austausch der Wappen, u.a. an den Stadttoren ${ }^{3}$. Später wurden auch die Fahnen der Zünfte eingezogen, wenn sie beispielsweise mit einem hessischen Wappen verziert waren ${ }^{4}$. Diese Schritte waren an sich nicht ungewöhnlich: Ende Januar 1806, als der preußische König die Herrschaft im ehemaligen Kurfürstentum Hannover übernommen hatte, waren die hannoverschen Wappen, falls sie nicht aus Stein waren, durch preußische Adler ersetzt worden ${ }^{5}$. Allerdings wurde ab 1807 die Abnahme der alten landesherrlichen Wappen und ihre Ersetzung zunächst durch den westphälischen Adler nicht immer mit äußerster Konsequenz bis in die hintersten Kantone des Königreichs Westphalen durchgeführt, denn, so Owzar, "gerade auf dem platten Lande stießen solche Maßnahmen immer wieder auf taube Ohren « ${ }^{6}$. Im Jahre 1811 versuchten lokale Autoritäten noch mit den administrateurs in Kassel zu argumentieren, ob es wirklich nötig sei, die alten beständigen Abzeichen vollständig aus ihrem Stadtbild zu tilgen ${ }^{7}$. Und

1 Napoleon, zitiert nach: Boudon, Le roi Jérôme, S. 313.

2 Vgl. SMidT, SiebeneICKer, Zur Ausstellung, S. 22.

3 Vgl. u.a. GoECKE, Das Königreich Westphalen, S. 6. Die provisorische Regierung vor Ankunft des Königs Jérôme in seinen Landen hatte bspw. das alte Siegel mit dem hessischen Löwen noch beibehalten. Vgl. ibid., S. 7.

4 Vgl. KEIM, »Savoir vivre«, S. 133. Im vorigen Kapitel B V. (Zinngießer Taberger) wurde im Rahmen des Exkurses über den Umgang der Westphalen mit Tod und Sterben auch die Fahne einer Bruderschaft erwähnt, die einen Eingriff der Polizei auslöste.

5 Vgl. [MierZINSKY], Erinnerungen aus Hannover, 1843, S. 23.

6 OWZAR, Eine Nation auf Widerruf, S. 62 f.

7 Vgl. GStA PK, V. HA, Nr. 698, Briefecopiebücher der Verwaltung der Hohen Polizei zu Kassel, 28. 10.1811-März 1812: Schreiben Nr. 3908 von J. F. M. de Bongars, Generalinspektor der Gendarmerie mit der Hohen Polizei beauftragt, an W.-C. von Gossler, Präfekt in Halberstadt, Saaledepartement, 28. 12.1811; vgl. ferner StA MR, Best. 76a Nr.156, Akten der Präfektur des Fuldadepartements die Abnahme der Wappen vorheriger Landesherrn und die Aufstellung neuer Königlich-Westphälischen betr., 1808: Schreiben Nr. 452 von J. J. Siméon, 
selbst noch im März 1813 meldete Polizeiagent Eskuchen, dass einige Städte die gesamte westphälische Herrschaft über unbekümmert ihre alten Wappen, beispielsweise an den Stadttoren, beibehalten hätten ${ }^{8}$. Dennoch lag den westphälischen Herrschaftsvertretern viel daran, den Machtwechsel sichtbar zu machen und es wird ihnen wohl nicht entgangen sein, »dass zwischen symbolischem und aktivem Widerstand ein gewisser Zusammenhang bestand " ${ }^{9}$. Eine Art Denkrevolution sollte eigentlich über die Entfernung der alten Herrschaftsinsignien eingeleitet werden, die den Westphalen den Wandel der Zeit nachdrücklich beweisen sollte und auch im Entfernen von überkommenen Herrschaftsabzeichen wie dem Pranger als Leibstrafengerät zum Ausdruck kam, die nicht mit dem neuen Verfassungsstaat und seinen Prinzipien zu vereinbaren waren. Die Abschaffung der Leibstrafengeräte stand in einer Linie mit der Staatsrhetorik, die sich etwa in den Reden der Staatsräte vor den Reichsständen äußerte. Dort sprach Karl August Malchus von Marienrode vom »Staat ohne Vergangenheit «" ${ }^{10}$, während Siméon »die früheren Völker«, die nun dem westphälischen Staat angehörten, beglückwünschte, »in einem `Staat` zusammengefasst worden [zu sein], was Stärke und Reichtum erhöhe, die Barrieren bei Kommunikation und Kommerz fallen lasse und eine `Nation« schaffe $«^{11}$. Offenbar gehörte es 1813 wie-

Justiz- und Innenminister, an den Grafen A. von Hardenberg, Präfekt in Kassel, Fuldadepartement, 12.3. 1808; ibid., Rundschreiben vom Grafen A. von Hardenberg an die Ober-Wege-Kommission in Kassel und an die Unterpräfekten in Fritzlar und Paderborn, Fuldadepartement, 14.3. 1808; ibid., Schreiben Nr. 2592 von Ganzer, Oberingenieur, Mitglied der Baukommission in Kassel, an G. J. G. A. von Reimann, Präfekt in Kassel, Fuldadepartement, 2.9.1811; ibid., Schreiben vom Kantonsmaire Gudensberg, Fuldadepartement, an G. J. G. A. von Reimann, 11. 7. 1811; ibid., Schreiben von G. H. Kappel, Maire in Karlshafen, Fuldadepartement, an G. J. G. A. von Reimann, 19. 8.1811; ibid., Schreiben Nr. 10664 von G. J. G. A. von Reimann an Lenzmann, Maire in Beverungen, Fuldadepartement, 2. 9. 1811; ibid., Schreiben Nr. 11120 von G. J. G. A. von Reimann, an den Domänendirektor, 4.9.1811; ibid., Schreiben Nr. 454 vom Kantonsmaire in Fritzlar, Fuldadepartement, an G. J. G. A. von Reimann, 29. 9. 1811. Vgl. OwZAR, Eine Nation auf Widerruf, S. 63 f.; SUNDERBRINK, Experiment Moderne, S. $130 \mathrm{f}$.

8 Vgl. RNB St. Petersburg, F 993 Arch. Westf., K. 16, Nr. 9818-9849, hier Nr. 9835: Rapport Nr. 29 von C. E[skuchen], Polizeiagent, an J. F. M. de Bongars, 3. 3. 1813.

9 OWZAR, Eine Nation auf Widerruf, S. $64 \mathrm{f}$.

10 Über die Losung des »Staates ohne Vergangenheit« vgl. KLEINSCHMIDT, Geschichte des Königreichs Westfalen, S. 176. Vgl. auch Cramer, Geschichte des Königreichs Westphalen, S. 179f., 191, 266.

11 Siméon, zitiert nach: PETRI, Der Moniteur Westphalien, S. 193; vgl. auch Moniteur Wesphalien, Nr. 84, 9. Juli 1808, S. 338-344. Eine andere Ansicht über den tatsächlichen Stellenwert der Losung "Staat ohne Vergangenheit «, vertritt Knauer, vgl. KNAUER, »Der Zukunft Bild sei die Vergangenheit«, S. 532 f. Die Rhetorik der Reden aus der Zeit der Französischen Revolution ist besonders gut untersucht worden, vgl. SchliebEN-LANGE, Traditionen des Sprechens; DIES., Schriftlichkeit und Mündlichkeit; HUNT, The Rhetoric of Revolution. Vgl. ferner PABST, »À la lanterne!«. 
derum auch zu den ersten Maßnahmen der ins Königreich Westphalen einmarschierenden russischen Militärs, die französischen und westphälischen Adler zu beseitigen ${ }^{12}$. Vor diesem Hintergrund überrascht es also nicht weiter, dass bei den ersten Vorboten der Destabilisierung der westphälischen Herrschaft auch deren Wappen ins Visier ihrer Kritiker gerieten.

Der Austausch der Herrschaftswappen zwischen 1806 und 1813 lässt die Funktion der Herrschaftsinsignien als symbolbeladene Objekte, als so genannte »Semiophore«, erahnen ${ }^{13}$. Während mit den Karikaturen ein grundsätzlich und meist offen kritisches Medium untersucht wurde, wird im Gegenzug nach der Annahme und Nutzung der westphälischen Wappen als Wahrzeichen der westphälischen Herrschaft durch die Westphalen gefragt. Durch ihre ostentative Annahme oder demonstrative Ablehnung der Wahrzeichen brachten diese vermutlich auch ihre Akzeptanz oder Ablehnung der neuen Herrschaft zum Ausdruck. Es liegt nahe, aus dem Umgang der westphälischen Bevölkerung mit den Herrschaftsabzeichen auf ihre politische Orientierung zu schließen. Daher soll im Folgenden näher untersucht werden, inwieweit und in welcher Form die westphälische Bevölkerung sich mit den neuen Herrschaftsabzeichen identifizierte oder diese zurückwies.

\section{Wappen als Identifikationsangebot für die Westphalen und ihre Aneignung}

Die westphälischen Herrschaftsvertreter setzten den westphälischen Adler oder gar den französisch-kaiserlichen Adler beinahe inflationär ein. Daher fehlte es für die Westphalen nicht an Angeboten, sich mit den Wappen der neu eingeläuteten Ära zu identifizieren ${ }^{14}$. Ein zeitgenössischer Jurist und Steuerinspektor berichtet im Jahre 1814 über die prunkvollen Zeremonien, die anlässlich des Eids der Deputierten auf König Jérôme am 1. Januar 1808 in Kassel stattfanden, dass die westphälischen Nationalfarben und Wappen, aber auch diejenigen des Kaisers Napoleon gleichermaßen gut vertreten ge-

12 Vgl. RNB St. Petersburg, F 993 Arch. Westf., K. 16, Nr. 9760-9796, hier Nr. 9763 : Rapport eines Polizeiagenten an J. F. M. de Bongars, [4].4. 1813.

13 Vgl. u.a. BARThes, Mythologies; POMIAN, Histoire culturelle. Über die Kongruenz von Symbolik und Sprache bei der Entfernung von Hoheitszeichen und beim Verlesen von Proklamationen vgl. SEverin-BARboutie, Für das »Vaterland «, S. 194.

14 Über die napoleonische Herrscherpräsentation unter Bezugnahme auf antike Referenzen vgl. der Tagungsbeitrag von L. Morenz, »Zwischen Adler und Biene. Napoleonische Herrschaftslegitimation in alten Spuren«, gehalten auf der Tagung "Antike(n)rezeption um 1800« am 10.-11.02. 2006, durch das Forschungszentrum Gotha für kultur- und sozialwissenschaftliche Studien der Universität Erfurt organisiert, in: H-Soz-u-Kult, [10.3.2006], http://hsozkult.geschichte.huberlin.de/tagungsberichte/id=1073 (9.6.2012). 
wesen seien ${ }^{15}$. Dieser Jurist scheint durch die besondere Erwähnung der kaiserlich-französischen Wappen- und Nationalfarben bei den zeremoniellen Feierlichkeiten auf die mangelnde Staatssouveränität des Königreichs Westphalen hinweisen zu wollen ${ }^{16}$. Der Stellenwert, der diesen staatlichen Attributen durch die Obrigkeit eingeräumt wurde, war offensichtlich beachtlich. Die ostentative Verwendung der Wappen lässt vermuten, dass die Herrschaftsvertreter sich ihrer Instrumentalisierung sehr bewusst waren.

Als sich im Jahre 1811 ein neuer westphälischer Adel organisierte, wohl auch, um die zweifelhafte adlige Herkunft einiger zugewanderter französischsprachiger Höflinge zu überprüfen, wurden die Mitglieder des alten und des neugeschaffenen Adels gebeten, als Wahrzeichen des westphälischen Adels zwei Fahnen mit dem westphälischen Adler in ihre Wappen aufzunehmen, die als gekreuzte Lanzen unter dem Wappenschild liegen sollten, berichtet der ehemalige Page von König Jérôme, Karl August Unico von Lehsten-Dingelstädt, in seinen Erinnerungen ${ }^{17}$.

Aber nicht allein die Deputierten und der Adel huldigten dem westphälischen Adler oder mussten ihn annehmen. Der Polizeiagent Cerfy berichtet über die eigentümliche Art der Bäcker in Homberg, mit dem westphälischen Wappen umzugehen. Anlässlich einer lokalen Festlichkeit zu Ehren des Königs, dessen Ankunft in der Stadt bevorstand, eigneten sie sich das Wahrzeichen des neuen Staates an, indem sie es buken:

Den 18 [März 1813] haben die Bürger zu Homberg eine Mahlzeit zu samen gehabt, wo auch die Gesundheit Sr. Majestet gedrunken wurde. -

Die Handwerker alda habe Sr. Majestet dem Nehmliche Tag auch erwartet, und sind auch, den nehmlichen Tag, bis in der Nacht, mit Musique in der Stadt herumgezogen, wobey, jedes Handwerk, eine fahnen mit dem Westphälischen Adler mit getragen hat, und auf der ander Seite ihre Handwerks wappen,

die Beker aber, haben einen Adler aus Teig gepaken, welcher sehr köstlich war, und an ihre fahnen oben angefährt ${ }^{18}$.

Die mögliche zweideutige Interpretation dieses Adlers aus Teig fiel dem Polizeiagenten offenbar gar nicht auf, der neben seiner Tätigkeit als Spitzel auch noch den Adler mitverspeiste: $\mathrm{Zu}$ dieser späten Zeit der westphälischen

15 Vgl. Cramer, Geschichte des Königreichs Westphalen, S. 76f.; SCHÜTT (Hg.), »Ein Mann von Feuer und Talenten«.

16 Im Online-Kapitel über die Gerüchte konnte ebenfalls als Teilbefund ermittelt werden, dass bereits vor 1813 das Thema der Staatssouveränität anhand des Verhältnisses ihres Monarchen zu seinem brüderlichen Gebieter die Westphalen beunruhigte, http://halshs.archives-ouvertes.fr/PLCI-NAPOLEON (1.1.2013).

17 Ein königliches Dekret vom 10. September 1811 regelte den neuen westphälischen Adel: Vgl. [LEHSTEN-DingELSTÄDT], Am Hofe König Jérômes, S. 61. Napoleon hatte diese Überprüfung der Herkunftsverhältnisse der französischsprachigen Höflinge herbeigeführt.

18 RNB St. Petersburg, F 993 Arch. Westf., K. 16, Nr. 9760-9796, hier Nr. 9775: Rapport von C[erfy], Polizeiagent in Kassel, an J. F. M. de Bongars, 20.3. 1813. 
Herrschaft das Wappentier zu verzehren, könnte eventuell auch einen verdeckten Hieb auf den kurz vor seiner Auflösung stehenden Staat darstellen.

Zumindest wäre es nicht das erste Mal gewesen, dass der westphälische Adler nicht ernst genommen wurde. Die Hinweise aus dem Quellenmaterial zeigen, dass spöttisches und höhnisches Verhalten im Umgang mit den westphälischen Wappen durchaus verbreitet war.

\section{Hohn und Spott auf die westphälischen Wappen}

So wurde der westphälische Adler im Allgemeinen als »westphälischer Kuckuck« verhöhnt, was wahrscheinlich auf seine schmächtige Statur zurückging, aber vielleicht auch auf die Abhängigkeit von Napoleon und die angezweifelte Souveränität des westphälischen Staates anspielte ${ }^{19}$. In den Versen Schellers werden die westphälischen Wappen mit »welschen Vögeln« verglichen:

Das Schloss sollt' werden umgebaut,

Und drum gezogen ein Stakkit

Mit Welschen Vögeln in der Mitt',

Die die Heraldik Aigles nennt,

Und die man als Stossvögel kennt ${ }^{20}$.

Auf einer der in Kassel beliebten Maskeraden fiel dem Polizeiagenten Henry Würz im Februar 1813 »ein Israelit Nahmens Koppel [auf,] welcher einen Haarbeutel an seiner Peruque befestiget hatte, worauf ein Adler gemacht war welcher sich in seine Flügel biß ${ }^{21}$. Mit diesem karnevalistischen Hinweis auf den westphälischen oder französisch-kaiserlichen Adler wollte der Träger

19 PRÖHLE, Die Fremdherrschaft, S. 11; vgl. ferner KLEINSCHMIDT, Geschichte des Königreichs Westphalen, S. 143; PetiTEAU, Portée de la politique symbolique, S. 150 .

20 SCHELlER, Jeromiade, S. 113. Falls die hier hergestellte Konnotation Allgemeingültigkeit im Königreich Westphalen hatte, ist bemerkenswert, dass die Staatsräte auf einer Maskerade am Hof allesamt als »welsche Hühner« erschienen, um auf ihre Unmündigkeit anzuspielen, so der Page Lehsten-Dingelstädt: »Eine bittere Satire legten die Mitglieder des Staatsrats an den Tag. Vielleicht um die Unwichtigkeit ihrer Stellung an den Tag zu geben, erschienen sie als Herde dindons (welsche Hühner), geführt vom Minister als Hirten. Denn nicht allein des Königs Kabinettsbefehl annullierte häufig ihre Beschlüsse, sondern sogar kaiserliche Gewalteingriffe hoben ihren Einfluß auf, machten sie zu dindons«. [LEHSTENDiNGELSTÄDT], Am Hofe Königs Jérôme, S. 52. Diese Satire könnte allerdings auch im Zusammenhang mit einem anderen »inneren Bild « der Westphalen stehen: Karikaturen und zeitgenössische Kommentare verglichen die Deutschen in ihrem Nationalcharakter häufiger mit einer »race moutonnière«. ZINSERLING, Denkwürdigkeiten, S. 28.

21 RNB St. Petersburg, F 993 Arch. Westf., K. 16, Nr. 9882-9987, hier Nr. 9919: Rapport Nr. 32 von H. W[ür]z, Polizeiagent in Kassel, an J. F. M. de Bongars, 8. 2. 1813. Der geschwächte Adler, dem die Federn ausgezupft werden, ist ein beliebtes Karikaturmotiv, vgl. L'anti-Napoléon, S. $77 \mathrm{f}$. 


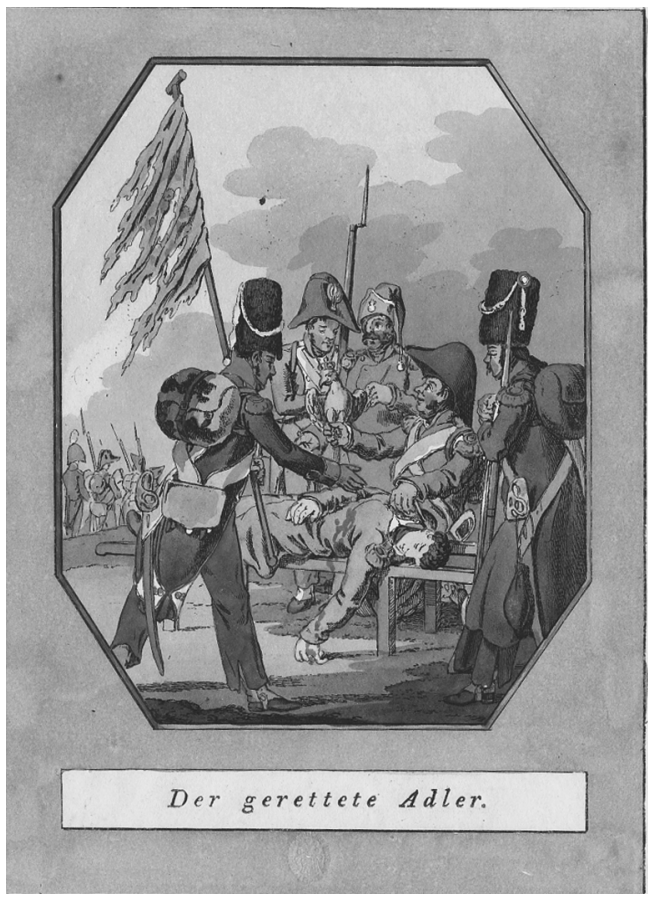

Abb. 28: C. G. H. Geißler,

Der gerettete Adler,

um 1810, Radierung,

$17 \times 21,5 \mathrm{~cm}, S M L$,

Gei III/47. Mit seiner Radie-

rung ironisierte der Zeichner

Geißler wohl die Überbewer-

tung des Adlers als Requisit

und Symbol eines jeden

französisch-kaiserlichen Regiments - ein Soldat stirbt

zwar, aber das von Napoleon seinen Truppen eingefleischte Symbol ist gerettet ${ }^{22}$.

womöglich auf den an den eigenen Ambitionen gescheiterten Eroberungswahn Napoleons abzielen.

Der westphälische Adler löste allerdings nicht immer nur feinsinnige, zweideutige oder dezent-humorvolle Reaktionen aus. Der Kasseler Polizeidiener Est erstattete im März 1813 Bericht über einen Zwischenfall, der ihm und seinem ehemaligen Kollegen Pfeil im Wirtshaus »Zum letzten Heller« zugestoßen war:

[Ich] ging [...] in die Stube des [...] Wirths $<$ Hohguth $>$ um mir von demselben eine Pfeiffe gegen Bezahlung zu holen, als ich mir nun solche von ihm ausboth, erwiederte mir derselber (:indem er meine Knöpfe am Rocke besahe) du hast Ja einen Adler auf Deinen Knöpfen auf diese $<$ scheiße $>$ ich aber Etwas. Du wirst im Arsch getreten ${ }^{23}$.

Die Zeugen dieses Austausches, nämlich Ests Begleiter Pfeil, Kaufmann Heinrich Krug, dessen Sohn und noch eine weitere Person wurden daraufhin auf den genauen Wortlaut der Verleumdung hin befragt. Kaufmann Krug senior leugnete, den angeblichen Ausspruch des Wirts Johannes Hoh-

22 Vgl. Petiteau, Portée de la politique symbolique, S. 148, $150 \mathrm{f}$.

23 RNB St. Petersburg, F 993 Arch. Westf., K. 21, Nr. 13 019-13 040, hier Nr. 13 031: Deposition Nr. 1 von Est, Polizeidiener in Kassel, 18.3. 1813. 
guth gehört zu haben ${ }^{24}$. Sein Sohn, Daniel Krug, zeigte sich einsichtiger und bestätigte, das Gespräch bezüglich der Knöpfe, des Adlers und des Hinterteils Ests gehört zu haben. Allerdings machte er nachträglich eine bedeutsame Einschränkung seiner Aussage. Hohguth habe dem Polizeidiener Est ausschließlich gesagt: "wer einen Adler auf den Knöpfe habe, daß ein solcher aus der Thür geworfen werde, und mußte er seine obige Erklärung: du wirst in den Arsch getreten zurücknehmen $\aleph^{25}$. Der Wirt selbst betonte in seinem Verhör, es sei lediglich wegen der Pfeife und des Tabaks zu einem Streit gekommen sei, weil Est ihm erst seine noch im Mund steckende Pfeife habe entwenden wollen und daraufhin sich eine solche hinter dem Ofen hervorgeholt habe, um sie mit dem Tabak des Wirtes zu stopfen. Außerdem bestritt er etwas vom Adler oder gar von Fäkalien gesagt zu haben ${ }^{26}$. Die Eskalation von anfänglichem Spott auf den westphälischen Adler zu derberen Angriffen weist darauf hin, dass sich hinter der Leichtigkeit des Spottes eine durchaus ernste Kritik der Verhältnisse verbarg.

\section{1809: Ab- und Anmontieren westphälischer Wappen}

Als am 6. Mai 1809 Schill mit sechs seiner Schillianer in Hadmersleben im Elbdepartement eingeritten kam, improvisierten die Einwohner kurzerhand für sie ein Volksfest im Gasthaus in der Post: »An der Post war während dieser Zeit der preußische Adler wieder aufgestellt ${ }^{27}$.

Der herbeigeeilte Superintendent erkundigte sich prompt, ob das Fest dem französischen Sieg der Schlacht bei Regensburg vom April 1809 gelte, das vorgeschrieben war und noch ausstand. Mit der provokativen Antwort, mit der man ihn abspeisen wollte, konnte er nicht recht zufrieden sein, musste jedoch gute Miene zum bösen Spiel machen und abwarten »bis die Husaren weggeritten waren, dann nahm der plötzlich eine andere Miene an. Das Volk wurde ermahnt nach Hause zu gehen, der preußische Adler musste wieder dem westphälischen weichen $\aleph^{28}$. Als erste Handlung im Rahmen ihrer improvisierten Feierlichkeiten ersetzten die Hadmerslebener das west-

Ibid., Nr. 13 033: Verhörprotokoll von D. Krug, 18. 3. 1813.

26 Ibid., Nr. 13 036: Verhörprotokoll von J. Hohguth, 19.3. 1813. Ähnlich ordinäre Aussprüche versicherte der Sergent Kroschke beim inoffiziell gefeierten Jahrestag des Gefechtes zu Ölper zwischen dem Herzog von Braunschweig-Oels und den westphälischen Truppen am 1. August 1809 gehört zu haben. Vgl. RNB St. Petersburg, F 993 Arch. Westf., K. 5, Nr. 2613-2655, hier Nr. 263: Rapport von Kroschke, Sergent, an J. F. M. de Bongars, 1.8. 1812.

27 PRÖHLE, Die Fremdherrschaft, S. 18.

28 Ibid. 
phälische durch das preußische Wappen. Pröhle führt seine Geschichte mit dem um vier Jahre zu früh wieder angebrachten preußischen Wappen fort:

In Hadmersleben war nach jenem Abzuge der Schill'schen Reuter der Preußische Adler noch auf vier Jahre versteckt. >Abwarten hatte das der Superintendent genannt. Nun wurde er am 14. November 1813 bei dem Siegesfeste für die Schlacht bei Leipzig von einem alten Soldaten aus dem siebenjährigen Kriege einhergetragen ${ }^{29}$.

Nicht nur, dass es für bessere Zeiten versteckt aufbewahrt wurde, falls es "anders « werden sollte: Das preußische Wappen nahm einen zentralen Platz im Fest ein, das die Hadmerslebener anlässlich der Schlacht bei Leipzig organisierten. Sein Träger war ein alterfahrener Soldat, der bereits im Siebenjährigen Krieg für das preußische Wappen gekämpft hatte - ein performativer Akt ersten Ranges ${ }^{30}$.

\section{Demonstrativer Angriff auf die königlich- westphälischen Wappen an Herrschaftsgebäuden im Jahr 1813}

War das westphälische Wappen zunächst bloß Spott und Hohn ausgesetzt, so begannen im Jahre 1813 massive Angriffe auf die Herrschaftsinsignien ${ }^{31}$. Zumindest wurden mehrfach Fälle aus den Kantonen nach Kassel gemeldet, in denen sich Staatsbürger meist heimlich und nächtlich mit der Beschmutzung der westphälischen Wappen bemerkbar machten.

Von seiner Erkundungsreise nach Niedenstein im Fuldadepartement meldete der Polizeiagent Cerfy nach Kassel: »Zu grosen Ritter habe ich bemerket, daß der Namen Sr. Majestät J. N. samt der Krone, bis auf der untere Schrift befliesentlich aus gelöschet ist, wann ich nicht irre, mit gelbes Erde, überstrichen, dieses ist an dem Haus des Ortes Maire alda angehäftet ¿ $^{32}$.

Die Beschmierung mit Erde und Schlamm ereignete sich auch andernorts, so in Münden nahe Kassel, ermittelte Polizeikommissar Haas in einer ähnlichen Angelegenheit. Am 2. März 1813 meldete Haas dem Generalinspektor Bongars in Kassel: "Dans la nuit passée on a couvert de couleur noire tous les aigles royeaux devant les maisons des commis aux exercices

29 Ibid., S. 26.

30 Weiterführend vgl. DÜDIng, Friedemann (Hg.), Öffentliche Festkultur; KNAU$\mathrm{ER}$, Tableaux patriotiques«.

31 Allerdings ereignete sich ein ähnlicher Fall, der ganz öffentlich stattfand und bei dem die Hallenser Bürger die westphälischen Abzeichen niederrissen und mit Kot bewarfen, bereits infolge des Schill'schen Zuges durch das Königreich Westphalen Anfang Mai 1809. Vgl. KLEINSCHMIDT, Geschichte des Königreichs Westfalen, S. $265 \mathrm{f}$.

32 RNB St. Petersburg, F 993 Arch. Westf., K. 16, Nr. 9760-9796, hier Nr. 9776: Rapport von C[erfy], Polizeiagent in Mission in Niedenstein, Fuldadepartement, an J. F. M. de Bongars, 18.2.1813. 
des 4 portes de la ville, l'aigle devant le bureau des droits de consommation et l'affiche devant la douane ${ }^{33}$. Bongars verbarg in seiner heftigen Antwort sein Entsetzen über den Vorfall durchaus nicht:

des malveillans ont poussé l'audace jusqu'à couvrer d'une couleur noire les armes royales apposées à differens endroits de la ville de Münden. Cette impudence est inouie, mettez tout en œuvre pour arriver à la decouverte des malveillans qui ont osé se porter à un exés de cette nature, Il faut agir aussi secretement que possible, car peut être ces scelerats se vandront eux memes pour vanter leur prouesse faites tout au momen pour les connoitre et si vous y parvenez arretez les sur le champ, saisissez vous de ses papiers et dirigez les sur Cassel ${ }^{34}$.

Haas antwortete ihm bald darauf, wie sein Verdacht in dem Fall lag, und teilte ihm mit, welche Maßnahmen er sich zur Ermittlung der Beschmierer vorstellte:

J'ai fait toutes les recherches possibles, pour decouvrir les personnes qui ont couvert de couleur noire les armes royales. [...] Tout cela a été sans succes. [...] Il n'y a que le nommé Sauer commis aux exercices \& le cordonnier Hagemann qui seroient capables de commettre de tels exces. Cependant on ne leur peut rien prouver. Ces deux hommes sont revenus chez eux à deux heures, le dernier a été gris, \& ce qui augmente encore mon soupçon c'est qu'on n'a pas oublié les armes d'aucune maison habitée d'un agent des contributions indirectes.

En consequence on peut croire que quelqu'un qui connoit toutes ces maisons ait été parmis ces scelerats. Probablement celui ci a été un agent des droits indirects, car les autres habitans ne connoissent pas toutes les maisons pourvues d'aigles.

Tout cela me donne le soupçon que le nommé Sauer agent des contributions indirectes ait commis avec ses consortes ces exces, mais je n'ai pas pu reussir de pouvoir le prouver.

En cas que le nommé Sauer l'auroit fait les nommés Utermöhlen, Eckhard et les autres personnes suspectes, s'ils ne sont pas ses complices, en sont au moins informés.

Avant hier j'ai reussi de trouver un agent qui me paroit être fait, pour penetrer les desseins nuisibles de ces personnes. C'est un vitrier nommé Grüne, qui connoit tres bien les personnes en question. [...] Je ne doute plus que quelques liaisons dangereuses avec ces personnes et le Maire Thomas ayent lieu ${ }^{35}$.

Als Anhaltspunkt für seine Recherchen diente Haas die Überlegung, der oder die Täter müssten dem westphälischen Staat als Steuerbeamte verpflichtet sein, da diese zielgerichtet alle Häuser auffinden konnten, die mit

33 RNB St. Petersburg, F 993 Arch. Westf., K. 17, Nr. 10 356-10 399, hier Nr. 10388 : Schreiben Nr. 96 von Haas, Polizeikommissar in Münden, Fuldadepartement, an J.F. M. de Bongars, 2.3.1813.

34 RNB St. Petersburg, F 993 Arch. Westf., Nr. 13 850: Registre de correspondance du Bureau de la police secrète (2.1.-18.4.1813), hier Schreiben Nr. 532 von J. F. M. de Bongars an Haas, 3. 3. 1813.

35 RNB St. Petersburg, F 993 Arch. Westf., K. 17, Nr. 10 356-10 399, hier Nr. 10 386: Schreiben Nr. 97 von Haas an J.F. M. de Bongars, 5.3.1813. Einzelheiten der diversen Ermittlungen von Haas gegen die Personen Utermöhlen, auch Utermühlen genannt, und Sauer finden sich im Online-Kapitel über das Medium »Brief«, http://halshs.archives-ouvertes.fr/PLCI-NAPOLEON (1. 1. 2013). 
dem westphälischen Wappen gekennzeichnet waren. Bongars schärfte ihm in seinem Rückschreiben ein, den Fall mit der allerhöchsten Diskretion zu behandeln ${ }^{36}$.

Haas stellte lediglich über den Rapport seines Geheimagenten fest, die Besudelung der westphälischen Wappen gehöre tatsächlich zu den Gesprächsthemen der Verdächtigen, die sich über seine ins Leere laufenden Ermittlungen belustigten:

En arrivant à Wilhelmshausen le nommé Hagemann, [...] dans l'auberge, [... a parlé des armes royales qu'on a couvertes de couleur noire, \& en riant il a dit, - le commissaire de Police m'a suspecté, mais pour mon bonheur ma femme n'etoit pas au logis, quand il a voulu l'interroger ${ }^{37}$.

Auf diese Anzeige hin baute Haas folgende Überzeugung auf:

Maintenant je suis persuadé plus que jamais que les nommés Sauer \& Hagemann sont les auteurs de l'exces par rapport aux armes royales. [Le but serait d'] apprendre si les hommes suspects sont effectivement dans des liaisons dangereuses ou s'ils ne font que des sottises sans but ${ }^{38}$.

Die Verdächtigten wurden letztlich jedoch in diesem Fall nicht überführt, dafür wies ihnen Haas nach, dass sie für die Verbreitung einer so genannten russischen Proklamation verantwortlich waren und sowohl Utermöhlen als auch Sauer kamen zur Bestrafung von April bis Juli 1813 in das Kasseler Staatsgefängnis ${ }^{39}$. Möglicherweise war Bongars nicht gewillt, öffentlich preiszugeben, dass selbst Herrschaftsvertreter in Handlungen gegen ihren Staat involviert waren und es erschien passender, sie wegen eines anderen Vergehens ins Kastell von Kassel zu überführen.

Auch in Celle wurde Anfang März 1813 ein Vorfall gemeldet, bei dem die westphälischen Wappen, diesmal am dortigen Postamt und bei dem Kaufmann Vieth mit Schlamm bedeckt wurden:

[L']enseigne au bureau de la poste, portant les armes Royales, ainsi que celle chez le marchand Vieth, chargé du dépôt de la poudre à tirer, ont été toutes deux pen-

36 Vgl. RNB St. Petersburg, F 993 Arch. Westf., Nr. 13 850: Schreiben Nr. 564 von J. F. M. de Bongars an Haas, 6.3.1813.

37 RNB St. Petersburg, F 993 Arch. Westf., K. 17, Nr. 10 356-10 399, hier Nr. 10372 : Schreiben Nr. 122 von Haas an J. F. M. de Bongars, 22. 3. 1813.

38 Ibid.

39 Vgl. RNB St. Petersburg, F. 993 Arch. Westf., K. 16, Nr. 9327-9383, Affäre Sauer und Utermöhler; vgl. RNB St. Petersburg, F 993 Arch. Westf., K. 11, Nr. 58225832; RNB St. Petersburg, F 993 Arch. Westf., Nr. 13 850: Schreiben Nr. 270 von J. F. M. de Bongars an Haas, 7.2. 1813; ibid., Schreiben Nr. 295 von J. F. M. de Bongars an Haas, 9. 2. 1813; ibid., Schreiben Nr. 396 von J. F. M. de Bongars an Haas, 17.2. 1813; RNB St. Petersburg, F 993 Arch. Westf., Nr. [13 852], Registre des personnes arrêtées, Einträge Nr. 171, 172 und 291. Über die vermeintlichen russischen Proklamationen vgl. ISKJUL', Russische Flugblätter. 
dant la nui, couvertes de boue, que l'auteur de cette vile action n'a, malgré toutes les recherches, pu etre decouvert encore ${ }^{40}$.

Bongars empfahl auch hier, die Untersuchungen mit größter Vorsicht $\mathrm{zu}$ führen ${ }^{41}$. Mancherorts wurden anstatt von Schlamm und Erde, Leim und Gips verwendet: Im Kern blieb die Aussage ähnlich, bis auf den Unterschied, dass Leim und Gips nicht so einfach von den Herrschaftsvertretern entfernt werden konnten ${ }^{42}$.

Die Empörung Bongars' zeigt, dass die westphälische Obrigkeit eigentlich nicht gewillt war, solche Angriffe zu dulden. Als im Jahr 1806 General Joseph Lagrange, Regent in Kassel, im Auftrag von Napoleon einen hessischen Aufstand, angeführt von Hauptmann Ludwig Thielo von Uslar und von Leutnant Junck von Kirchhain, niederschlug, soll Napoleon ihn angewiesen haben, ganz besonders den Aufständischen aus Eschwege und Hersfeld folgendes zu übermitteln: "Man soll ihnen meinen Willen kundgeben, dass die Beleidigungen, welche meinen Adlern zugefügt worden sind, nur durch Blut gerächt werden können « ${ }^{43}$. Der Ausspruch, ob wahr oder imaginär, zeigt, wie emotional aufgeladen die Angelegenheit war und wie treffsicher die Bürger die Machthaber reizen konnten, wenn sie sich an den obrigkeitlichen Zeichen und ihrer Rhetorik vergriffen. Die Westphalen mit ihren späteren Attacken auf die westphälischen Herrschaftsabzeichen griffen den Staat an einem wunden Punkt an, nämlich an seinen Insignien als herrschaftseigenen rhetorischen Argumenten.

Berücksichtigt man, dass das nächtliche Beschmieren der westphälischen Wappen ab dem Frühjahr 1813 an verschiedenen Orten im Königreich Westphalen erfolgte, so wird deutlich, dass dieses Vorgehen entweder eine gewisse Öffentlichkeit über das weiterverzweigte Informationsnetzwerk erlangte und zur Wiederholung an anderen Orten anregte oder dass die Aktion der Besudelung und Entweihung der landesherrlichen Abzeichen bereits vor 1807 zum festverankerten Handlungsrepertoire der westphälischen Bevölkerung gehört hatte ${ }^{44}$. Da die Wappenbeschmierungen vielerorts fast zeitgleich stattfanden, kann man davon ausgehen, dass die Einzelerscheinungen in einer alten Tradition aus vorwestphälischer Zeit standen.

40 RNB St. Petersburg, F 993 Arch. Westf., K. 17, Nr. 10 494-10 686, hier Nr. 10604 : Schreiben Nr. 487 von F. T. de Guntz, Generalpolizeikommissar der Hohen Polizei in Braunschweig, an J. F. M. de Bongars, 2.3.1813.

41 Vgl. RNB St. Petersburg, F 993 Arch. Westf., Nr. 13 850: Schreiben Nr. 569 von J. F. M. de Bongars an F. T. de Guntz, 6.3.1813.

42 Vgl. KEIM, »Savoir vivre«, S. 133.

43 Napoleon, zitiert nach: GOECKE, Das Königreich Westphalen, S. 23, vgl. S. 15-22.

44 Vgl. AlgaZI, Kulturkult, S. 113 f. 


\section{Weitere Eskalation in den Angriffen der Westphalen auf die westphälischen Wappen}

Abgesehen von den exemplarisch erwähnten Fällen, bei denen die Wappen nur beschmutzt wurden, eskalierte andernorts die Gewalt, wobei die Gegner der westphälischen Herrschaft die Wappen sogar beschädigten oder zerstörten.

So wurden beispielsweise in den Kommunen Brackede, Blackede und Hitzacker im Elbdepartement Ende März 1813 die westphälischen Adler heruntergerissen, als ihre Einwohner von der angeblichen Ankunft der Russen auf dem rechten Elbufer erfuhren ${ }^{45}$. Ebenfalls bewirkten im Oktober 1813 in der Gemeinde Rössing im Ockerdepartement einige »Individuen ", die wohl trotz aller Bemühungen des Unterpräfekten in Hildesheim nicht mehr vor Auflösung des Königreichs Westphalen ermittelt werden konnten, die Entfernung von königlichen Wappen an sämtlichen staatlichen Häusern $^{46}$.

In Bergen im Allerdepartement wurden nachts Steine gegen die Fenster eines Steuerbeamten geworfen und die westphälischen Wappen, die am Postamt angebracht waren, niedergerissen ${ }^{47}$. Die Schuldigen wurden allerdings überführt, da sie offenbar im Affekt gehandelt und ihre Aktion nicht ausreichend getarnt hatten, wie es bei den Fällen der Beschmutzung der westphälischen Wappen üblich war $^{48}$. Über das Entstehen von Exzes-

45 Vgl. GStA PK, V. HA, Nr. 741, Briefcopiebücher des Generalkommissars der Hohen Polizei Moisez in Sachen der geheimen Polizei, 16. 9. 1812-27.7.1813: Schreiben Nr. 621 PS. von Moisez, Generalpolizeikommissar der Hohen Polizei in Halberstadt, an J. F. M. de Bongars, 25.3.1813; RNB St. Petersburg, F 993 Arch. Westf., Nr. 13 850: Schreiben Nr. 795 von J. F. M. de Bongars an J. F. C. von Düring, Unterpräfekt in Uelzen, Allerdepartement, 29.3. 1813. Vgl. ferner SPEITKAMP, Unruhe, Protest, Aufstand, S. 142. Vgl. das Online-Kapitel über die Gerüchte, http://halshs.archives-ouvertes.fr/PLCI-NAPOLEON (1.1.2013); auch diese besaßen durchaus in bestimmten Situationen die Macht, die Bevölkerung zu Handlungen, Tätlichkeiten und Übergriffen zu verleiten. Vgl. u.a. FARGE, La vie fragile; FARGE, REVEL, Logiques de la foule; FARGE, Rumeur, ville et roi.

46 Vgl. HStAH, Hann. 52, Nr. 140, Anzeigen von aufrührerischen Bewegungen und Tumulten, 1813, Bl. 3 f.: Schreiben von G. A. von Nordenflycht, Unterpräfekt in Hildesheim, Ockerdepartement, an G. A. von Wolffradt, Innenminister, 20. 10. 1813.

47 Auch andere westphälische Beamte wurden 1813 mit Steinwürfen gegen ihren Amtssitz, der zugleich ihr Privatwohnsitz war, konfrontiert. Nicht nur westphälische Beamte waren von solchen Vorfällen betroffen: Die Beherrschung der französischen Sprache und der Umgang mit den Douaniers waren bereits ausreichend, um Opfer der Steinewerfer zu werden, vgl. KAHMANN, Die Geschichte des J. F. A. Lampe, S. 342.

48 Vgl. RNB St. Petersburg, F 993 Arch. Westf., K. 13, Nr. 7494-7519, hier Nr. 7494: Schreiben Nr. 519 von F. T. de Guntz an J.F. M. de Bongars, 6.3.1813; RNB St. Petersburg, F 993 Arch. Westf., Nr. 13 850: Schreiben Nr. 612 von J. F. M. de Bongars an F. W. Frantz, Präfekt in Hannover, Allerdepartement, 9. 3. 1813; vgl. 
sen und die Eskalation von Gewalttätigkeiten beim Zusammenbruch der westphälischen Herrschaft lieferte Birgit Hoffmann bereits in einer Untersuchung einige Überlegungen, die deren tradierte Muster aus vorwestphälischer Zeit deutlich machen und die Täter überwiegend unter Jugendlichen feststellen ${ }^{49}$. An Steinwürfe auf ihre Amts- und Privatsitze waren die lokalen Herrschaftsvertreter offensichtlich gewöhnt: Während des DörnbergAufstands in Felsberg im April 1809 hatte der Maire vorsichtshalber seine Fenster weit aufgesperrt, damit kein Glas zu Bruch gehen konnte ${ }^{50}$. Die Angabe des Pfarrers Gehren darüber macht deutlich, dass solche Angriffe meist eher als Drohgebärden mit allenfalls materiellem Schaden zu bewerten waren.

Auf die gewalttätigen Übergriffe reagierten einige der lokalen staatlichen Vertreter, indem sie vorbeugend selbst die Wappen der durch sie vertretenen Herrschaft entfernten. Solche Vorkehrungen empörten dann die Vorgesetzten in Kassel ganz besonders:

le maitre de poste à Neustadt a oté les armes de Westphalie placées sur le devant de sa maison et les a remplacé par un tableau portant pour toute inscription: Poststation, parce que des deserteurs et autres mauvais sujets venus des environs de Nienbourg avoient jetté des pierres contre les armes de Westphalie pendant plusieurs nuits du dernier clair de lune.

Je ne puis me dispenser de vous denoncer la puisillanimité de ce Maitre de poste, qui est d'autant plus evidente qu'il etoit bien le maitre de reclamer l'assistance de la police et de la Gendarmerie pour être protegé contre toute insulte. Il se pourroit aussi qu'il eut eu encore d'autres motifs pour se defaire si promptement des armes du royaume ${ }^{51}$.

Das Rechtfertigungsschreiben des Postmeisters zu Neustadt, in dem er an Bongars' Verständnis appelliert und von seiner Verzweiflung und Angst spricht, entlastete ihn allerdings nicht von der Verdächtigung durch den Generalinspektor der Gendarmerie ${ }^{52}$. Im obigen Quellenzitat wird auch der

auch HStAH, Hann. 52, Nr. 3026, Verschiedenes Material der Hohen Polizei, 1810-1813, Bl. 120: Schreiben von J. F. M. de Bongars an F. W. Frantz, 9. 3. 1813; ibid., Bl. 121: Schreiben von K.W.A. von Stralenheim, Unterpräfekt in Celle, Allerdepartement, an F. W. Frantz, 11.3.1813.

49 Vgl. HoffmanN, Aufrührer, Ruhestörer oder gute Patrioten? Über Lokalbeamten als Zielscheibe kollektiver Gewalt vgl. BRAKENSIEK, Fürstendiener - Staatsbeamte - Bürger, S. 350. Brakensiek hat für die Unruhen in Grebenstein im Jahre 1813 seinerseits bewiesen, dass die gewaltsamen antiobrigkeitlichen und antisemitischen Proteste in den Jahren 1819, 1830/31 und 1848 in der Tradition der »Insurrektionen gegen die Fremdherrschaft« standen, vgl. DERS., Strukturen eines antinapoleonischen Aufstandes, u.a. S. 61, ferner S. 52.

50 GeHren, Dreimalige Verhaftung und Exportation, S. $12 \mathrm{f}$.

51 RNB St. Petersburg, F 993 Arch. Westf., Nr. 13 850: Schreiben Nr. 747 von J. F. M. de Bongars an A. J. F. Pothau, Generalpostdirektor in Kassel, 24. 3. 1813; vgl. ferner ibid., Schreiben Nr. 746 von J. F. M. de Bongars an F. W. Frantz, 24. 3. 1813.

52 Vgl. ibid., Schreiben Nr. 782 von J. F. M. de Bongars an F. W. Frantz, 28. 3. 1813; vgl. Almanach royal de Westphalie, 1811, S. 257. 
Angriff auf die westphälischen Abzeichen eindeutig als »insulte«, Beleidigung oder Verleumdung, eingestuft. Den Angriff auf die westphälischen Wappen rückten die Polizeibehörden in die Nähe einer Verleumdung gegen den Staat, nicht weit vom crime lèse-majeste ${ }^{53}$.

Dabei wird die Entscheidung einiger westphälischer Staatsbeamter, sich der westphälischen Wappen voreilig zu entledigen, verständlich, wenn man sich ihrer Perspektive annähert, so über folgendes Schreiben, das zwar nicht über Vorkommnisse aus dem Königreich Westphalen selbst berichtet, jedoch leicht auf die westphälischen Verhältnisse übertragen werden kann. Der Sohn eines Maires aus dem Siegdepartement im Großherzogtum Berg schreibt zu Anfang des Jahres 1813:

Dans quelle position difficile et embarassente ne sommes nous pas placés, nous autres fonctionnaires allemands, sous le gouvernement du grand Empereur des Français!

Ciel! c'est avec l'âme navrée de douleurs et les larmes aux yeux que je vous rends compte, Monsieur le sous-préfet, des scènes tumultueuse qui ont eu lieu hier et cette nuit, et dont nous sommes encore menacée.

Une bande de gens mal intentionnés des environs de Hambourg, Ronsdorf, Sohlingen etc., arriva hier au soir à Gummersbach, ainsi que vous en aurez déjà été informé. [...] une masse de 500 hommes, se rendit à Neustadt pour y assassiner les gendarmes et les douaniers, qui heureusement s'étaient sauvés. [...]

Ciel! c'est toi que je prends à témoin de mes souffrances. Oú me sauverai-je avec ma femme malade et mes malheureux enfants. Je n'entends que les cris: (ce sont des rimes allemandes de la populace) un, deux, trois, le tirage finit à la fois. Un, deux, trois, quatre, les russes vont être ici. regie, Régie, Kylogramme, Kylogramme, nous sommes les maitres et c'est nous qui gouvernons: qui n'est pas pour nous perira par nous. Tous les fonctionnaires seront mis à mort.

Jugez de la sensation que celà doit produire. Quelques brâves gens pourraient, en s'opposant à main armée à ces devastateurs, rétablir la tranquilité; mais oú sont-ils? Tous crie: vive les Russes, les Français sont des brigands. [...]

Je suis le fils du maire Wenche, qui écris le présent en son nom. Mon père s'est sauvé; [...] Si seulement mon bon père et ma malheureuse mère étaient en sureté $!^{54}$

53 Bereits in den Kapiteln über das Medium »Brief« (Online-Kapitel: http://halshs. archives-ouvertes.fr/PLCI-NAPOLEON [1.1.2013]) und Kapitel B V. (Zinngießer Taberger) wurden Beispiele genannt, die zeigen, wie die Staatsverleumdung und der crime lèse-majesté in der Fantasie der Westphalen fest verankert waren. Insgesamt interessieren sich Historiker weniger für diese inhaltlich minderwertigen >Auswürfe`, die dennoch ebenso von den kulturellen und kommunikativen Praktiken der Zeitgenossen zeugen. Weiterführend vgl. u.a. DINGES, Ehrenhändel; HOHKAMP, »Auf so ein erlogenes Maul gehört eine Maultaschen«; RUBLACK, Anschläge auf die Ehre; REUTER, Der König verdient kein Denkmal; LINGELBACH, Injurie und Injuriensachen; PETITEAU, Violence verbale.

54 AN Paris, 29 AP 39, Lettres confidentielles du comte de Nesselrode, ministre de l'Intérieur du Grand-duché de Berg, 1807-1814, Bl. 85: Schreiben von Wenche, Sohn des Maires von Neustadt, an den Unterpräfekten in Siegen, Siegdepartement, 29.1.1813. Auch allerorts im Königreich Westphalen waren die Maires 1813 durch die Angrifflust der städtischen bzw. ländlichen Bevölkerung sehr besorgt. Vgl. BRAKENSIEK, Strukturen eines antinapoleonischen Aufstands, S. 60. 
Die Verzweiflung ist nicht zu verkennen, die Tränen und die Aussichtslosigkeit werden ebenfalls erwähnt und die Lieder der aufgebrachten Bevölkerung mit der Drohung, den Staatsvertreter zur Rechenschaft ziehen zu wollen, sind inhaltlich wiedergegeben. Der Ernst der Lage konnte sich offensichtlich schnell verändern und die aufgebrachte Bevölkerung wirkte auf den Sohn des Maires unberechenbar. Die Beschreibung macht deutlich, vor welcher Art von Ausschreitungen sich die westphälischen Beamten schützen wollten, wenn sie von selbst die westphälischen Wappen entfernten.

Nach der Phase der oben genannten Identifikations- und Aneignungsrituale in Bezug auf die westphälischen Wappen, die in einer der Herrschaft huldigenden Geste entstanden, tauchen in den Polizeiakten ab dem Jahr 1813 zunehmend Vorkommnisse auf, in denen sich Staatsvertreter von den westphälischen Wappen distanzieren oder diese gar, auf Selbstschutz bedacht, eiligst ablegen. So musste Stadtwachtmeister Welger in Schmalkalden bereits im Januar 1813 daran erinnert werden, er habe unverzüglich die ihn ausweisende westphälische Kokarde wieder zu tragen ${ }^{55}$.

Parallel zu den immer häufigeren Nachrichten vom dramatischen Rückzug aus Russland und vom Eindringen der Kosaken ins Innere des Königreichs Westphalen ereigneten sich auch mehr Übergriffe auf die westphälischen Wappen oder Distanzierungen der westphälischen Staatsvertreter von den herrschaftlichen Abzeichen.

Der Angriff auf die westphälischen Wappen blieb jedoch nicht ausschließlich ein Mittel der aufgebrachten westphälischen Bevölkerung: Aus der Nähe von Celle wurde im März 1813 gemeldet, ein Kommando von 30 französischen Soldaten habe die westphälischen Wappen mit ihren Gewehrkolben zerschlagen ${ }^{56}$. Der genaue Hintergrund ist leider schwer nachvollziehbar, zeigt jedoch, dass es verfehlt wäre, den Angriff der Westphalen auf die westphälischen Wappen nur als einen patriotisch-nationalen Akt einzustufen ${ }^{57}$. Die Wappen wurden auch zum Streitobjekt zwischen der westphälischen und der französisch-kaiserlichen Regierung, da sie nicht nur für die innerwestphälischen Verhältnisse als Kommunikationsangebot galten.

55 Vgl. RNB St. Petersburg, F 993 Arch. Westf., Nr. 13 850: Schreiben Nr. 78 von J.F. M. de Bongars an F. T. de Guntz, 13. 1.1813.

56 Vgl. RNB St. Petersburg, F 993 Arch. Westf., K. 17, Nr. 10 494-10 686, hier Nr. 10 538: Schreiben Nr. 623 von F. T. de Guntz an J. F. M. de Bongars, 22. 3. 1813.

57 Als mögliche Erklärung könnte der Douanenstreit dienen, der im Laufe der westphälischen Herrschaft für zahlreiche Konfliktausbrüche und Vorfälle zwischen Frankreich und Westphalen sorgte, in den Jérôme persönlich eingriff und sich mit seinen Staatsbürgern solidarisch gegen Napoleon verbündete. Vgl. KLEINSCHMIDT, Geschichte des Königreichs Westfalen, S.311, 317, 322f. Vgl. einschränkend dazu BERDING, Napoleonische Herrschafts- und Gesellschaftspolitik, S. 17. 


\section{Schlussakt: Zerstörung des Napoleonstandbilds}

Ein anonymer Autor beschreibt im Jahre 1814, wie Jérôme in Kassel auf dem vom »Königs«- zum »Napoleon«- umgetauften Platz eine Napoleonstatue errichten ließ und macht sich lustig darüber, wie diese mitten in der Kartoffelblüte beim Wochenmarkt stand ${ }^{58}$. Tatsächlich beließen es die staatskritischen Westphalen anfänglich dabei, sich über die Napoleonstatue lustig zu machen und gestalteten dies mitunter mit Hilfe eines Spottgedichts über die Marmorstatue: "Zu Kassel auf dem Zaitenstock, ohne Hemd und ohne Rock, ohne Schuh' und ohne Hosen, steht der Kaiser der Franzosen « $^{59}$. Die Angriffe auf die Wappen ließen schon ahnen, dass Napoleon nicht lange nach der Auflösung des Königreichs Westphalen auf seinem Sockel stehen bleiben würde. Zunächst griffen die russischen Militärs die Statue an: »Die Kosacken haben später [...] der Statue die Nase und eine Hand [abgeschlagen] ${ }^{60}$, berichtet F. Müller und beschreibt auch, wie die Westphalen nach ihnen die Statue behandelten: »Ihre Barbarei ist aber auch noch überboten worden, als man mit andern Theilen des trefflichen Kunstwerkes den beschädigten Mantel des Landgrafen auf dem Friedrichsplatze stickte « ${ }^{61}$. Es besitzt hohe Symbolkraft, dass ausgerechnet die Reste des gestürzten Napoleonstandbildes zum Ausbessern der Statue des Landgrafen von HessenKassel verwendet wurden, die willkommene Rückübertragung der obrigkeitlichen Instanz auf die alte hessen-kasselanische Dynastie scheint jedenfalls damit bekräftigt worden zu sein.

\section{Umgang mit Wappen zwischen tradiertem Muster und ungekannter Eskalation}

Die Frage nach dem Umgang der Westphalen mit den Wappen ihrer Herrschaft zeigt eine Wandlung von der friedvollen Huldigung der Wappen und Herrscher in Form von gebackenen Wappen bis hin zum Sturz der Napoleonbüste auf dem Napoleonplatz in Kassel. In der Angriffs- und Gewaltbereitschaft der Westphalen gegenüber den Wappen konnte eine kontinuierliche Eskalation festgestellt werden - selbst wenn die Angriffe im Affekt erfolgten, so waren sie sehr wohl dosiert und erstaunlich kalkuliert. Immer häufiger und weiter verbreitet, wurden die Angriffe auf die Wappen zum Ventil für angestaute Frustrationen gegenüber der westphälischen Herrschaft. Die vielerorts auftretenden nächtlichen Beschmutzungen und Zerstörungen der königlich-westphälischen Wappen an Herrschaftsge-

58 Vgl. Anonymus, Die französische Garküche, S. 14; KiRCheIsen, König Lustig, S. 74. Die Napoleonstatue war auf Veranlassung eines königlichen Dekrets am 15. November 1812 errichtet worden. Vgl. LYNCKER, Historische Skizzen, S. 90.

59 Zitiert nach: MÜLLER, Kassel seit siebzig Jahren Bd. 1, S. 43.

60 Ibid.

61 Ibid. 
bäuden im Jahr 1813 sind sicherlich im Zusammenhang mit einer weiter zurückreichenden Tradition zu sehen. Aber selbst wenn man darin tradierte Praktiken zu erkennen glaubt, war es seitens der Westphalen keinesfalls belanglos, den westphälischen Staat an seiner stark artikulierten Staatssymbolik anzugreifen. Diese Strategien hatten einen demonstrativen und kommunikativen Charakter. Sie zielten darauf ab, in der Übergangszeit vor dem Ende der napoleonischen Ära weite Bevölkerungsteile zur Abkehr von der französisch-westphälischen Herrschaft zu überzeugen und zu mobilisieren. Sogar einige Staatsvertreter, die ihrer Loyalitätspflicht gegenüber dem westphälischen Staat hätten folgen sollen, waren in die Beschmutzung westphälischer Wappen verwickelt, wie dies mit dem vom Polizeikommissar gemeldeten Fall aus Münden angedeutet werden konnte. Dies zeigt, wie groß der Unmut gegenüber der westphälischen Herrschaft im Jahre 1813 bereits war und wie weitreichend die westphälische Bevölkerung ihrem Landesherrn das Vertrauen entzog.

Mit der Beschmutzung und der Zerstörung der landesherrlichen Abzeichen wurde ein letztes eindeutiges und sprachübergreifendes Signal an den "Fremdherren" gesandt, das ihn zum Rückzug bewegen sollte. Mit dem Übergriff auf die Wappen waren die deutsch-französischen Sprachbarrieren jedenfalls irrelevant.

Der antiwestphälische Protest, der sich gegen die Wappen artikulierte, erfolgte unter Aneignung eines Herrschaftsabzeichens: Das Kommunikationsangebot ihres Monarchen, der auf Zeremoniell und Prunk viel Wert legte, wurde durch die widerspenstigen Westphalen angenommen; die Raffinesse lag darin, dass man sich auf die rhetorisch argumentative Ebene der westphälischen Herrschaft begab, um diese zu kritisieren. Die Westphalen hatten die Staatsrhetorik verinnerlicht und in ihre "symbolischen Praktiken« integriert: Sie hatten die »Mikrotechniken der Politik» gelernt und kehrten diese 1813 nun gegen den Urheber dieser Symbolik ${ }^{62}$. Die Wappen sind somit als im Kommunikationsprozess der Westphalen und der Obrigkeit involviert zu verstehen und der Angriff auf sie ist als fest im Handlungsrepertoire eingegliedert zu betrachten. Die oben dargestellte Entwicklung beweist, dass König Jérôme mit seiner Herrschaftssymbolik bei den Staatsbürgern im Laufe seiner siebenjährigen Herrschaft bei der medialen Festlegung auf bestimmte Attribute sehr wohl erfolgreich gewesen war und dass die Mobilisierung gegen ihn im Jahr 1813 eben über die von ihm durchgesetzten rhetorischen und symbolischen Zeichen erfolgte ${ }^{63}$.

62 Hunt, zitiert nach: LANDWEHR, Geschichte des Sagbaren, S. 156.

63 Bereits in den Kapiteln B IV. (Karikaturen) und B V. (Zinngießer Taberger) konnten ähnliche Prädispositionen der herrschaftskritischen Staatsbürger, sich der Rhetorik und Symbolik der Herrschaft zu bedienen, um ihre Kritik zu verpacken, angedeutet werden. Vgl. ferner OWZAR, Der alte Schein des neuen Reiches, S. 160. 